\title{
Text Entry from Power Wheelchairs: EdgeWrite for Joysticks and Touchpads
}

\author{
Jacob O. Wobbrock, ${ }^{1}$ Brad A. Myers, ${ }^{1}$ Htet Htet Aung ${ }^{1}$ and Edmund F. LoPresti ${ }^{2}$ \\ ${ }^{1}$ Human-Computer Interaction Institute \\ School of Computer Science \\ Carnegie Mellon University \\ Pittsburgh, PA 15213 USA \\ \{ jrock, bam, hha \}@cs.cmu.edu \\ ${ }^{2}$ A.T. Sciences, Inc. \\ 160 N. Craig Street, Suite 117 \\ Pittsburgh, PA 15213 USA \\ (412) 687-1181 \\ edlopresti@acm.org
}

\begin{abstract}
Power wheelchair joysticks have been used to control a mouse cursor on desktop computers, but they offer no integrated text entry solution, confining users to point-and-click or point-anddwell with on-screen keyboards. But on-screen keyboards reduce useful screen real-estate, exacerbate the need for frequent window management, and impose a second focus of attention. By contrast, we present two integrated gestural text entry methods designed for use from power wheelchairs: one for joysticks and the other for touchpads. Both techniques are adaptations of EdgeWrite, originally a stylus-based unistroke method designed for people with tremor. In a preliminary study of 7 power wheelchair users, we found that touchpad EdgeWrite was faster than joystick WiVik, and joystick EdgeWrite was only slightly slower after minimal practice. These findings reflect "walk up and use"-ability and warrant further investigation into extended use.
\end{abstract}

\section{Categories and Subject Descriptors}

H.5.2 [Information Interfaces and Presentation]: User Interfaces - input devices and strategies, user-centered design

K.4.2 [Computers and Society]: Social Issues - assistive technologies for persons with disabilities

\section{General Terms}

Design, Experimentation, Human Factors

\section{Keywords}

Power wheelchair, computer access, joystick, touchpad, text entry, text input, unistrokes, gestures, EdgeWrite, Pebbles.

\section{INTRODUCTION}

People with motor impairments, such as those caused by Muscular Dystrophy, Cerebral Palsy, Parkinson's disease, or spinal cord injuries, often cannot use a conventional mouse and keyboard. They may lack sufficient mobility to reach for these devices, sufficient motor control to switch accurately and efficiently between them, or sufficient endurance to use them for

Permission to make digital or hard copies of all or part of this work for personal or classroom use is granted without fee provided that copies are not made or distributed for profit or commercial advantage and that copies bear this notice and the full citation on the first page. To copy otherwise, or republish, to post on servers or to redistribute to lists, requires prior specific permission and/or a fee.

ASSETS'04, October 18-20, 2004, Atlanta, Georgia, USA

Copyright 2004 ACM 1-58113-911-X/04/0010...\$5.00.

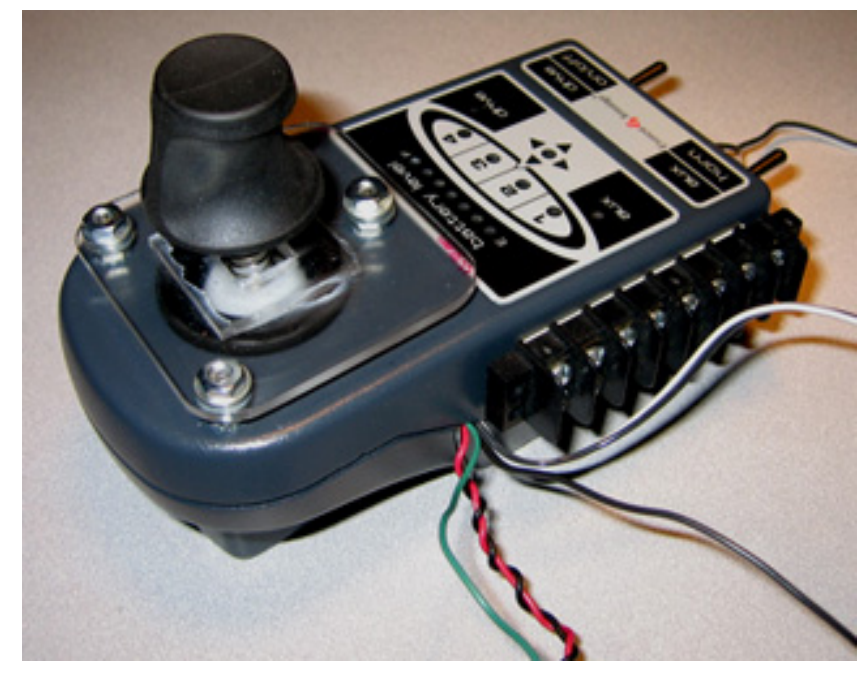

Figure 1. The Everest \& Jennings 1706-5020 power wheelchair joystick we modified for EdgeWrite text entry. Note the plastic template around the stick, which provides a square boundary.

more than a few minutes. In addition, many people with motor impairments use wheelchairs. An estimated 1.4 million Americans depend on wheelchairs for mobility [14]. About $10 \%$ of these people use power wheelchairs, and about half of them require more than one assistive technology to participate in daily activities [4]. A computer access solution that works with an existing device, rather than adding to the mix of encumbering devices, would be desirable [11]. Such solutions have been termed "integrated control systems" [27].

Commercial technology already exists for providing mouse cursor control from a power wheelchair joystick [28]. But mouse control is only part of a computer access solution. The ability to enter text is also a cornerstone of successful human-computer interaction. However, an integrated text entry method to accompany joystick mouse control is unavailable. Instead, text entry from power wheelchairs takes the form of point-and-click or point-and-dwell with on-screen keyboards. This exacerbates the need for window management due to decreased screen real-estate. It also imposes a secondary focus of attention, taking users' eyes from their work. A text entry method for power wheelchair joysticks would give fuller access without requiring additional devices (Figure 1).

Though less common than joysticks, touchpads can also be used to control power wheelchairs [29] (Figure 2). Touchpads require less strength to operate than joysticks and no calibration. The further a finger moves from the center of the touchpad, the faster the wheelchair moves. While touchpads have been studied and 


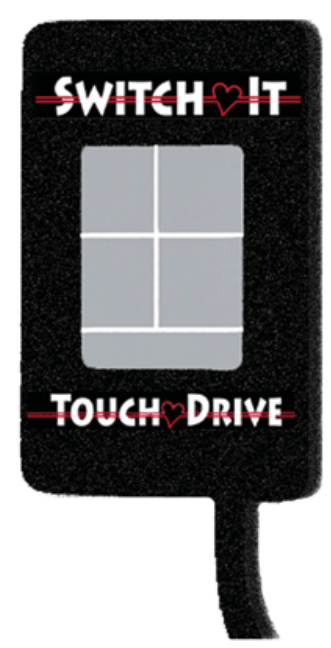

used extensively for mousing (e.g., [12,23]), they have not often been used for text entry. People in power wheelchairs might benefit from an integrated device that could control their chair, mouse, and text entry solution. This requires a versatile text entry technique for touchpads.

As more public information terminals (kiosks) appear in building lobbies and libraries, on streets, in subways, and in community centers, the ability to access these terminals becomes more important. Just as the Americans with Disabilities Act requires that many buildings have access ramps, future terminals may be required to be accessible electronically via Bluetooth or another wireless technology. It would be advantageous to have an integrated control system where the power wheelchair joystick or touchpad could be used as the input device for mousing and text entry for such terminals.

\subsection{Our Approach}

As a part of the Pebbles research project, we are investigating how handheld devices-broadly defined to include Personal Digital Assistants (PDAs), joysticks, touchpads, and other devices-can be used concurrently with desktop computers [19]. In our previous work [21], we showed that a Palm PDA could be effective for computer access for some people with motor impairments. This is because while many people with motor impairments lack gross motor control, strength, and endurance, they retain enough fine motor control and finger dexterity to negotiate the small expanse of a PDA screen. The same may be true for joysticks and touchpads.

In addition, we developed a new assistive text entry technique called EdgeWrite [33]. Originally for use with a stylus, EdgeWrite enables people with tremor and reduced mobility to write on a PDA, even though many of them cannot write Graffiti [2], the dominant unistroke alphabet for Palm PDAs. (In addition, EdgeWrite was over $18 \%$ more accurate than Graffiti for ablebodied users.) We also implemented a version of EdgeWrite for game controller joysticks, and found that able-bodied users were faster and produced more accurate text with it than with date stamp and selection keyboard, two prevalent joystick text entry methods [31]. Although game controller joysticks are different than power wheelchair joysticks, EdgeWrite is simple and versatile and can be adapted to a variety of devices.

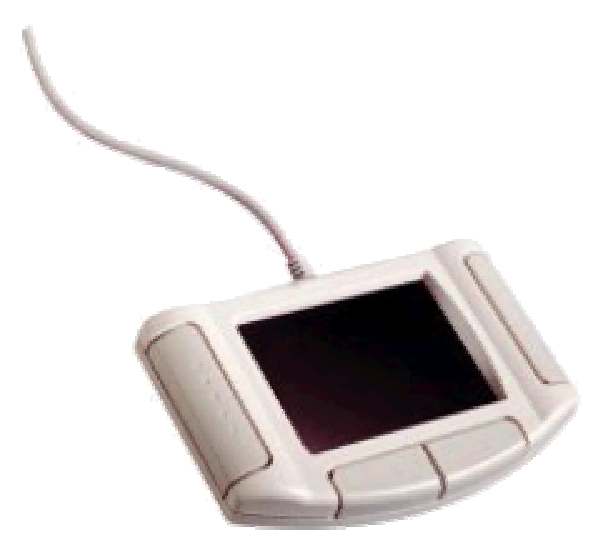

Figure 3. The Synaptics touchpad we used in our studies. Because the touchpad itself is rectangular and EdgeWrite requires a square, we imposed a plastic template over the touchpad surface (not shown).

For the current work, we redesigned EdgeWrite to work on an Everest \& Jennings power wheelchair joystick (Figure 1) and a Synaptics touchpad (Figure 3). We compared EdgeWrite on each of these devices to a commercially available text entry method accessible using a power wheelchair joystick or touchpad-the on-screen WiVik keyboard [22,25].

We conducted participatory design sessions with 7 power wheelchair users, 6 of whom had Cerebral Palsy and 1 who had Multiple Sclerosis. The participants entered text phrases using touchpad EdgeWrite, joystick EdgeWrite, and joystick WiVik and gave us feedback. Learning EdgeWrite gestures in a single session was difficult compared to learning the on-screen WiVik keyboard, which many participants had used before. Despite this, touchpad EdgeWrite was the fastest, joystick WiVik was second, and joystick EdgeWrite was a close third. The results were promising since participants had little time to learn the EdgeWrite alphabet before testing.

This study confirms that gestural text entry methods often take longer to learn than selection-based methods [31]. But a quality gestural method offers a number of advantages over selectionbased methods: it does not require precious screen real-estate, it can be used without looking, it can be customized (or "trained"), and it can require less motion per character, since gestures can be quite small but keyboards can only be shrunk so much before their keys are difficult to acquire.

Thus, this work takes a step toward a more complete integrated control system for computer access and wheelchair control by addressing the need for text entry from existent power wheelchair joysticks and touchpads.

\section{RELATED WORK}

Many devices exist for computer access, some of which can be used from a power wheelchair [1]. Alternative physical and onscreen keyboards, head switches, sip-and-puff devices, voice recognition systems, and augmentative communication devices are just a few of the options available for computer access. But there can be obstacles to effective deployment. Many devices are prohibitively expensive. Others require extensive configuration or maintenance. Some might be unwieldy, even on a power 
wheelchair. These and other reasons may be why prior work has found that less than $60 \%$ of people who indicate that they need adaptations actually use them [9]. Our aim in this work, by providing text entry techniques for existing power wheelchair control systems, is to lower the barriers to computer access by using mechanisms already present.

Stylus-based EdgeWrite is related to other unistroke text entry methods, most notably the original Unistrokes [10] and Graffiti [2]. Few methods besides EdgeWrite have been devised for "writing" with a joystick, but an interesting exception is myText, a commercial system for small mobile phone joysticks [5].

EdgeWrite depends on physical edges to provide high tactility and stability of motion. Other work has explored using edges in interaction techniques, such as placing controls along edges for easier target acquisition $[8,32]$. The classic Lisa and Macintosh user interfaces had their menus along the top edge of the screen for the same reason [16].

Mouse cursor control using a power wheelchair joystick has been recently studied $[15,24]$, but not with an integrated text entry technique. Like the current work, the second of these evaluations used the on-screen WiVik keyboard.

Touchpad interaction techniques have been around for some time, but surprisingly few text entry techniques exist for touchpads. Two exceptions are a touchpad used for a remote control [7] and for numeric entry using a clock-face metaphor [13]. Neither of these, however, is a generic touchpad text entry technique like EdgeWrite. Most touchpad techniques focus on control and selection tasks (e.g., [12,17,23]). Similar to EdgeWrite, templates have been used on touch surfaces to guide finger motion [3].

\section{EDGEWRITE DESIGN AND IMPLEMENTATION}

\subsection{Stylus EdgeWrite for PDAs}

EdgeWrite was originally designed as a stylus-based text entry method on PDAs for people with motor impairments, especially tremor, since Graffiti proved difficult for this population [33]. The properties of EdgeWrite and its alphabet (Figure 4) make it wellsuited to other devices for assistive text entry, like power wheelchair joysticks and touchpads.

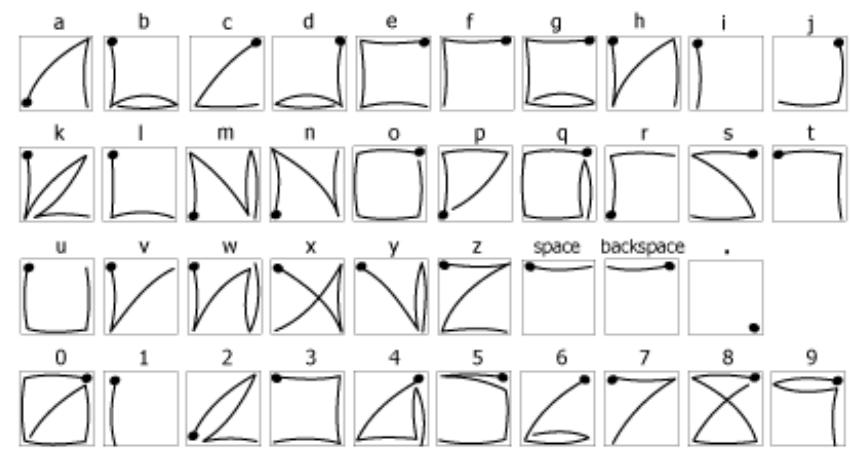

Figure 4. EdgeWrite letters and numbers. A full character chart is available elsewhere [33]. The bowed line segments between corners are for illustrative purposes only. All motion is in straight lines between corners.
Specifically, EdgeWrite relies on physical edges and corners to provide stability during motion [30]. A user moves his or her stylus, finger, or joystick along the physical edges and into the corners of a square bounding the input area. Recognition does not depend on the whole path of motion, but on the order corners are hit. This means that moderate wiggle and tremor do not deter good recognition. It also means that to add a custom gesture, a user only needs to perform it once, indicating the desired order of corner-hits. EdgeWrite is not a pattern-matcher and does not require a training set, so recognition occurs without ambiguity.

\subsection{Joystick EdgeWrite for Power Wheelchairs}

We implemented a version of EdgeWrite in C++ for the Everest \& Jennings 1706-5020 power wheelchair joystick, which was removed from its chair (Figure 1). Wires were attached to the joystick outputs and the left auxiliary switch in order to access the voltage signals corresponding to the absolute $(x, y)$ position of the stick and the state of the switch (Figure 5). A National Instruments 6024E DAQCard read the signals and made them available to our software.

The joystick was polled for its position every $5 \mathrm{~ms}$. When the $(x, y)$ position entered one of the four EdgeWrite corners, a character trace began. When the $(x, y)$ position returned to the center of the square for a short duration, the trace was deemed complete and recognition of the corner sequence occurred [31].

The joystick's coordinate plane was restricted to the square hole imposed by the template that bounded the stick. A design consideration was the size of this square (Figure 6). In our design iterations, we found that an edge length of $13.75 \mathrm{~mm}$ worked well. It was small enough to reduce the amount of necessary movement, but big enough to reduce the risk of accidental corner-hits. The template was mounted on three bolts which we installed from the underside of the joystick chassis.

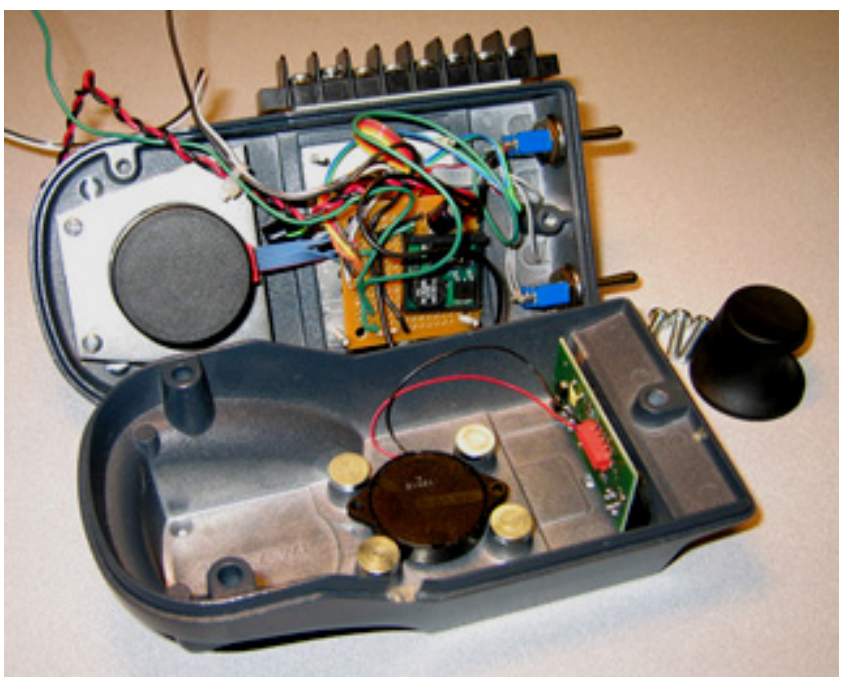

Figure 5. Inside the Everest \& Jennings joystick. We added wires to emit the $(x, y)$ joystick position and the state of a switch. 


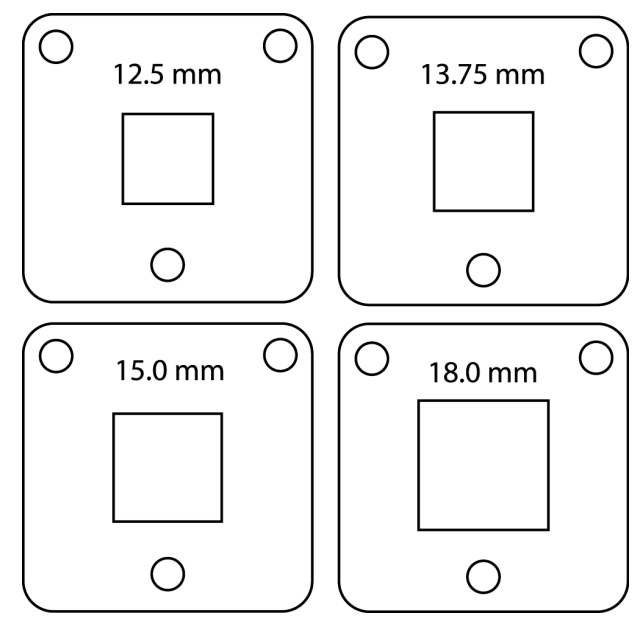

Figure 6. Diagrams of the plastic templates used to impose square bounding areas around the joystick. In pilot tests, the $13.75 \mathrm{~mm}$ square had the best balance between speed and accuracy, since smaller squares are faster but more prone to accidental corner-hits. The holes were used for mounting. (See also Figure 1.)
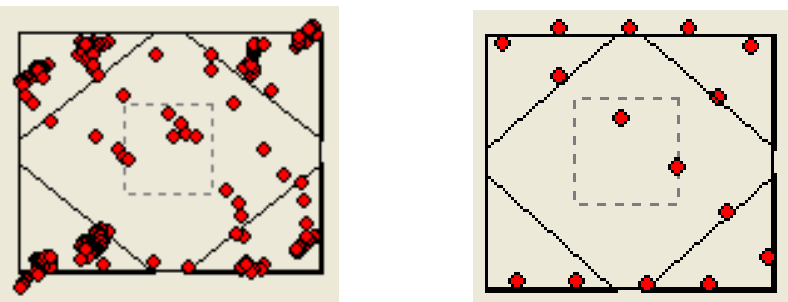

Figures 7a, 7b. An unfiltered (left) and filtered trace of " $s$ " with the joystick. Note the triangular corners and the dashed center box used for snap-to-center segmentation.

The $(x, y)$ position from the joystick was very noisy, in essence containing a great deal of electronic "tremor" (Figure 7a). To filter out this noise, we took the last $n$ points and computed a running average, treating the result as an individual point. Trialand-error yielded $n=12$ as the value that best removed sufficient noise while decreasing the inevitable lag introduced by the running average (Figure $7 b$ ).

\subsection{Touchpad EdgeWrite for Power \\ Wheelchairs}

We implemented another version of EdgeWrite in $\mathrm{C}++$ for a Synaptics touchpad (Figure 3). Like the stylus and joystick versions, the touchpad version used a plastic template to provide a square boundary (Figure 8). While joystick EdgeWrite was found to be highly sensitive to the size of the square boundary, the touchpad version was not; the square shown in Figure 8 was 30 mm wide and worked well.

Touchpad EdgeWrite is similar to stylus EdgeWrite in that letter segmentation is accomplished by using pen/finger up. Before a finger goes down on the touchpad, the corners are considered rectangular. Once a finger enters a corner area, however, the corners deflate into triangles, preventing diagonal strokes from accidentally hitting unintended corners (Figure 9).

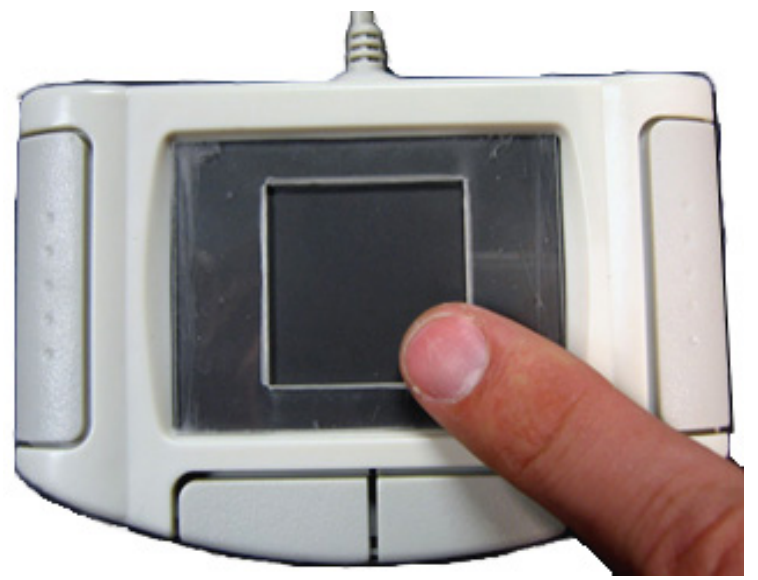

Figure 8. The Synaptics touchpad we used with a plastic template to create a square area for EdgeWrite text entry.

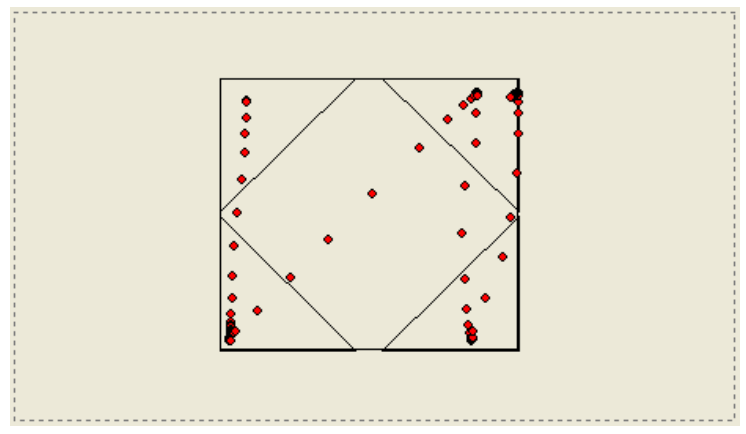

Figure 9. The trace of an EdgeWrite " $w$ " on the touchpad. The image maps to the whole touchpad surface, while the square in which the " $w$ " occurs maps to the template's square hole.

The edges of the touchpad's plastic template aid tremulous finger motion in the same way that they aid stylus motion on a PDA [33]. Users can feel the smooth plastic edges as they move, exerting pressure against them for stability and tactile feedback. Since the touchpad surface only senses human skin, pressure on the plastic template does not interfere.

\subsection{Expert Performance with Three Devices}

To appreciate the differences among EdgeWrite versions, an ablebodied EdgeWrite expert, this paper's first author, was given a text entry test using phrases from MacKenzie and Soukoreff [18]. He entered 10 phrases with each EdgeWrite implementation: the PDA stylus, the Everest \& Jennings joystick, and the Synaptics touchpad. His respective speeds were 23.0, 12.8, and 19.1 words per minute (WPM). His respective error rates were $6.2 \%, 8.4 \%$, and $11.4 \%$. All errors made during entry were corrected (though this hindered speeds), so these data represent perfect transcription. While this only reflects one expert, it gives a ballpark comparison consistent with other studies [31,33].

\section{PARTICIPATORY DESIGN AND EVALUATION}

This section describes our design and evaluation sessions with participants, in particular the lessons we learned and the parameters we identified. Throughout the process we worked closely with real power wheelchair users. 


\subsection{Mouse Control and the WiVik Keyboard} In order to compare EdgeWrite to a currently available means of text entry with a wheelchair joystick, we compared the EdgeWrite techniques described above to the on-screen keyboard WiViK $[22,25]$ accessed with the wheelchair joystick. We implemented proportional mouse control software in $\mathrm{C}++$ to enable cursor control from the Everest \& Jennings joystick. We also enabled a switch on the joystick to simulate a mouse click. When the switch was pressed, it enacted a mouse-down. When the switch was released, it enacted a mouse-up. Prior research [15] shows that among the possibilities for joystick-driven mouse control, a ratecontrolled approach is both fastest and most accurate for onscreen keyboard text entry, as opposed to absolute positioning or a hybrid design. The velocity and acceleration of our ratecontrolled implementation were comparable to those used in the prior research. When using the joystick with the WiVik keyboard, we removed the plastic template required for EdgeWrite.

We used the WiVik keyboard with the default settings, which included no spacing between the keys, no word prediction, and click-triggering of keys rather than dwell-triggering. The keyboard consumed the entire width and about $1 / 3$ of the height of a $1024 \times 768$ screen. We chose the WiVik keyboard because of its familiarity as a mouse-driven on-screen keyboard.

\subsection{Participants}

We improved the three techniques that we evaluated-joystick and touchpad EdgeWrite, and joystick WiVik-with the help of 7 power wheelchair users. (We initially had 8 participants, but one was too impaired to perform any of the techniques.) Six of the 7 were from the UCP of Pittsburgh and had Cerebral Palsy. The other participant had Multiple Sclerosis. The average age of the participants was 25.9 years, with a low of 21 and a high of 67 . Participants had been in wheelchairs for an average of 14.0 years, with a low of 3 and a high of 30 . Two of the 7 participants were male. All but one of them used a conventional QWERTY keyboard for text input, but all of them said that they could only do so for short periods before becoming fatigued. Two of the participants had used a PDA only a little, and the other 5 had never used one at all. None of the participants had ever used EdgeWrite with a joystick or touchpad.

\subsection{Procedure}

In order to involve participants in the design of the techniques, we had them practice each technique before entering a test phrase of about 30 letters. Practice consisted of entering each letter 4 times in a row with a given technique (e.g., "aaaa bbbb ... zzzz"). This took 25-35 minutes with each of the two EdgeWrite techniques, and about 10-20 minutes with WiVik, since there were no gestures or alphabet to learn. The whole test did not exceed 2 hours. All 7 participants used WiVik and joystick EdgeWrite, but only 4 participants used touchpad EdgeWrite because of personal time constraints. (Comparing the joystick data from these 4 participants to the other 3 participants shows similar results, suggesting that the touchpad results for all 7 participants would not have been substantially different.)

As shown in Figure 10, an EdgeWrite character chart was visible during the session. With the slow pace of practice and the limited endurance of participants, we did not want to unduly burden them

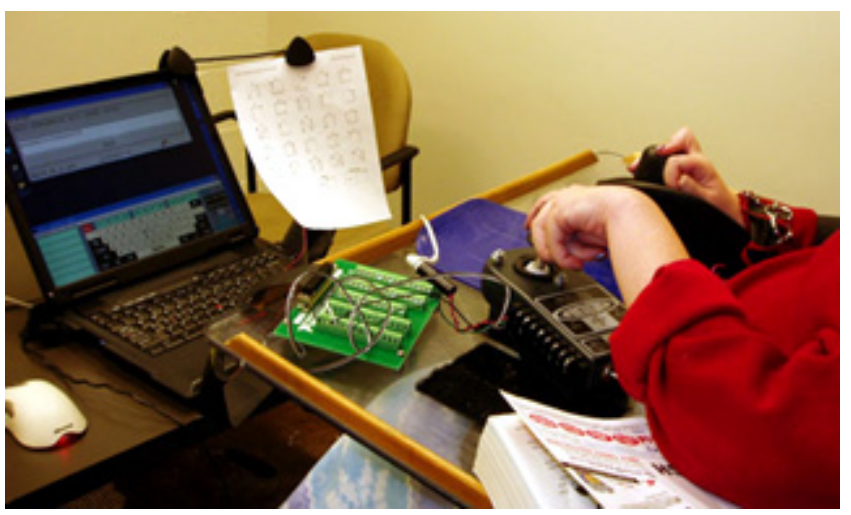

Figure 10. A participant entering text using the joystick and WiVik on-screen keyboard. An EdgeWrite character chart was on display for the entire study, even when unused in this condition.

with memorizing the EdgeWrite characters. Instead, we taught them how to read the chart and observed their behavior. However, reading the chart slowed them significantly compared to WiVik. The inter-character time - the time from the end of one character to the start of the next-suggests the delay caused by reading the chart. The average inter-character time was 6.23 seconds. With more practice, this value would go down, since participants would be familiar with the letters.

All text input was logged on the PC by our text entry test program. It was later analyzed with recently developed measures [26] that allow participants to enter text in an unconstrained, realworld fashion, where they can choose to fix errors or not. Participants were instructed simply "to proceed as quickly and accurately as possible."

We solicited responses from participants in between text entry phrases and more formally using questionnaires. In addition, many participants offered ideas while practicing with the techniques.

\subsection{Overall Results}

Practice sessions, slow performance, and eventual fatigue meant that we only had time for one test phrase by each participant with each method. Thus, we do not have sufficient data for statistical

\section{Average Words Per Minute}

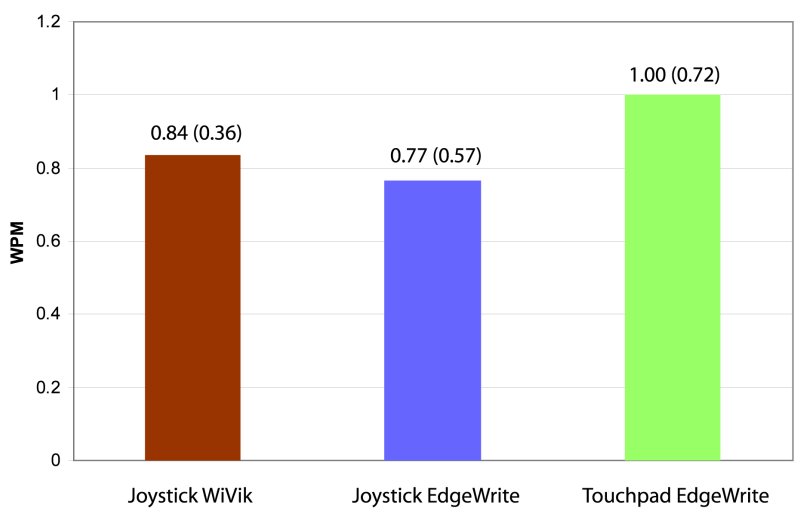

Figure 11. Average words per minute for each entry method. Higher values are better. Standard deviations are in parentheses. 
significance. However, we can compare means and correlate performance with participants' comments.

For speed, touchpad EdgeWrite was fastest on average at 1.00 (0.72) words per minute (WPM). Joystick WiVik was second at 0.84 (0.36) WPM. Joystick EdgeWrite was third at $0.77(0.57)$ WPM. These results are graphed in Figure 11.

Three error rates characterize unconstrained text entry. Corrected errors are those fixed during entry, uncorrected errors are those left in the transcribed string, and total errors are the sum of the two (Figure 12).

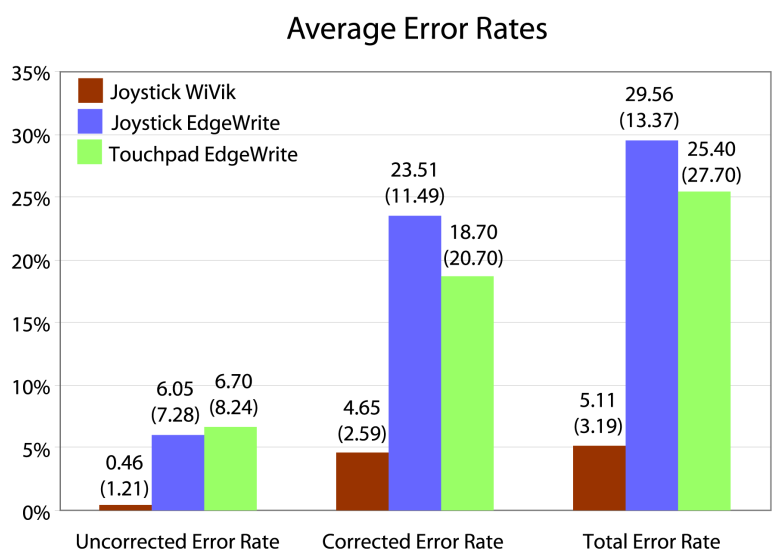

Figure 12. Average error rates for the three methods. Uncorrected errors appear in the final text, but corrected errors do not. Smaller values are better.

Clearly, participants made more errors with the EdgeWrite methods than with joystick WiVik. This is to be expected of a gestural input technique compared to a selection-based one, since when learning new gestures, users often perform them incorrectly. A longitudinal study would reveal whether this is merely due to unfamiliarity, or a problem that would affect practiced users. Note that to make an error with WiVik, a user must place the mouse cursor over the wrong key and still choose to press and release the switch, a multi-step operation that is readily avoided.

The questionnaire results showed that, of the 3 methods, participants felt the touchpad was easiest to use, easiest to learn,

\section{Subjective Ratings}

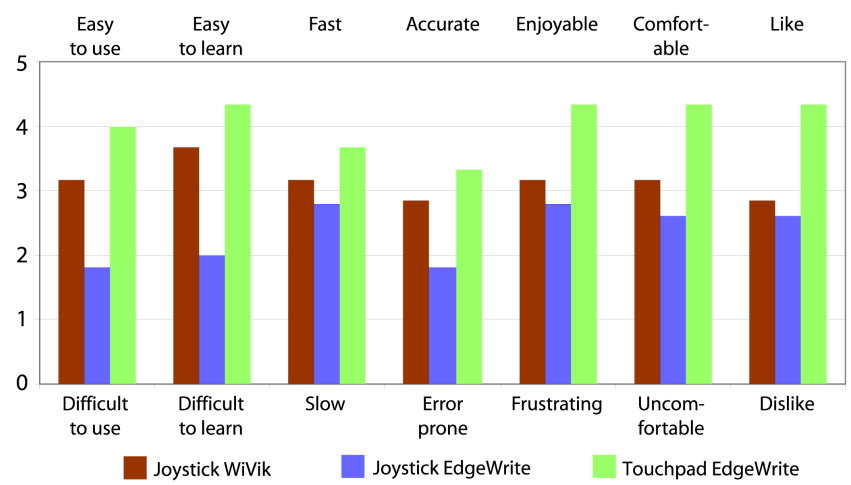

Figure 13. Average questionnaire ratings reveal a preference for touchpad EdgeWrite over joystick EdgeWrite and joystick WiVik. Higher values are better. fastest, most accurate, most enjoyable, most comfortable, and most liked overall. Participants rated joystick WiVik second in all of these categories, and joystick EdgeWrite third.

\subsection{Lessons from Participants}

Participant \#1 was a 67 year-old retired school teacher with Multiple Sclerosis. He was notable for two reasons: he was the only person without Cerebral Palsy, and he was only one of two participants who was faster with joystick EdgeWrite than joystick WiVik (1.91 vs. 1.22 WPM). The other was Participant \#8, who was a 22 year-old female with good fine motor control. She was only slightly better with joystick EdgeWrite than joystick WiVik (0.52 vs. 0.50 WPM). Participant \#1 showed us that the plastic template should be thicker to prevent the exposed spring on the joystick post from catching the template's edge. After using joystick WiVik for a few minutes he said, "It takes the patience of Job to do this." Upon switching from WiVik to EdgeWrite, he said, "I'm much faster with this; don't you think I'm much faster?" indicating his first impression of joystick EdgeWrite.

Participant \#2 was a 21 year-old student. She initially had trouble with the diagonal strokes with joystick EdgeWrite because she would move too slowly through the center, and EdgeWrite would try to recognize what she had already done. She motivated us to add the ability to change the center dwell time required for segmentation. If a polled joystick point falls outside the center area before the dwell time has elapsed, the dwell time counter resets. The time that worked well for Participant \#2 was $500 \mathrm{~ms}$. This participant also thought it would be easier to do the WiVik keyboard with the EdgeWrite template still on the joystick because it would help prevent target over-shooting. This suggests joystick mouse control and joystick EdgeWrite could co-exist on the same device without having to remove the EdgeWrite template.

A long dwell time was not sufficient for Participant \#4, a 40 yearold volunteer. She moved inconsistently with joystick EdgeWrite, sometimes making letters very quickly, other times pausing for many seconds to think. For her we added the ability to trigger recognition with the switch, removing the need for center dwell. She enjoyed touchpad EdgeWrite because it was the easiest method with which to fix mistakes. Of touchpad EdgeWrite she said, "Once you understand what you are doing, it goes completely well." Participant \#7 echoed this when she said, "If you get used to it, you'd be really fast I suppose."

While the females tended to interact too gingerly with the joystick, the males, Participants \#1 and \#3, were too forceful at first. Discovering the right speed and amount of pressure to exert against the joystick template was an important step in learning joystick EdgeWrite.

A common problem was that participants did not always start in the corner of the plastic template before making their gestures with the joystick. This was less of a problem with the touchpad. The reason may be that the joystick must be pushed from somewhere (i.e., the center) to reach the starting corner, whereas a finger can begin in the corner of the touchpad.

Participant \#4 gave us an important insight into the design of the touchpad template. We originally smoothed the edge of the touchpad template so that it was slightly beveled. But this caused participants' fingers to slip up onto the template's surface, 
actuating a "finger up" and prematurely triggering recognition. This insight led to the fabrication of a thicker touchpad template, the edges of which we left vertical and unbeveled.

Participant \#6 highlighted the importance of end-user customizability. While using touchpad EdgeWrite, this participant's finger did not always press against an edge of the square. Having defined the square exactly along the plastic edges, we realized that her fingers moved inside this square, and that the actual square in which she moved was therefore smaller. We then had her redefine the EdgeWrite square, and her accuracy improved tremendously.

Finally, the diagonal strokes were difficult for many users of joystick EdgeWrite. This is not surprising, because it is along the diagonals that the user does not have an edge to press against. The letter "k" (Figure 14a) was particularly problematic because of its two diagonals in a row. After the study, we designed a new form of " $k$ " (Figure 14b) that is still reminiscent of a Roman " $k$ " but without a diagonal. In later investigations, this new form of " $k$ " proved much easier to perform and became a permanent part of the alphabet in all versions of EdgeWrite.
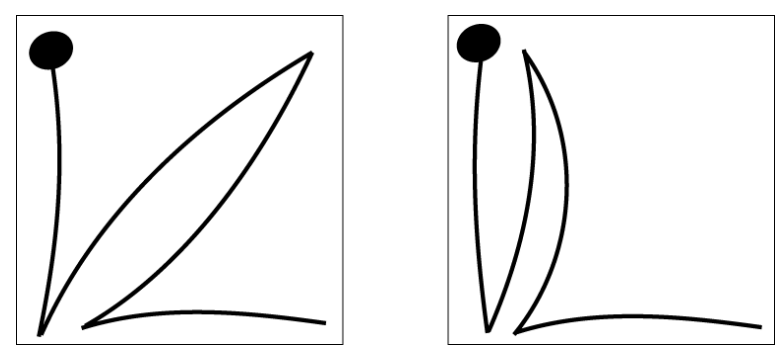

Figures 14a, 14b. The original design of the letter "k" (left) was difficult for joystick users because of the diagonal strokes. An alternate " $k$ " was later designed that contains no diagonals. This " $k$ " is much easier to make with a joystick. Note that arcs are only illustrative. Actual motion is in straight lines.

\section{FUTURE WORK}

The biggest limitation of this preliminary study is the relatively short time participants had to learn and practice EdgeWrite. While previous work shows that able-bodied users can learn EdgeWrite in about 15 minutes [31,33], participants with motor impairments require more time in which to accomplish the same amount of practice. We therefore want to do a longitudinal study in which participants are allowed time to become experts. Such a study would yield results for learning, retention, longevity, fatigue, and whether joystick or touchpad EdgeWrite meets participants' realworld text entry needs. As it stands, this initial study represents "walk up and use"-ability more than general usability. These results warrant further investigation and design refinement.

The success of input techniques depends largely on numerous physical and psychological factors, many of which can still be optimized for EdgeWrite, in particular the square size for the touchpad and spring strength, grip size, and height of the joystick. Prior work [6] shows large gains are possible with subtle optimizations like these.

Once the design is improved, the next step is to integrate mouse control into both devices and provide for switching among mouse, text, and wheelchair control. We can then study the integrated control system in a holistic fashion. For example, we could have participants move between terminals where they would do both mouse and text entry tasks. Other design issues arise here, for example, if we have more than one person controlling a terminal at a time. Techniques for coordinating multiple handheld interfaces to a single desktop computer have been explored [20] and could be employed.

\section{CONCLUSION}

We have described two means of integrating text entry into preexisting controls on power wheelchairs: one using a wheelchair joystick and the other a touchpad. Both devices are small, light, inexpensive, and require minimal configuration, giving them significant practical advantages as integrated control systems over dedicated computer access technologies. We described our designs and implementations of EdgeWrite and the participatory role real power wheelchair users played in our development process. While these techniques can still be improved, this work has paved the way for their future refinement, and ultimately, better computer access from power wheelchairs.

\section{ACKNOWLEDGEMENTS}

The authors would like to thank Janis Thoma-Negley and the UCP of Pittsburgh, Steve Hayashi, John A. Kembel, Rich Simpson, John Springsteen, A.T. Sciences for the joystick, and Elaine Wherry and Synaptics, Inc. for the academic touchpad kit.

This work was supported in part by grants from the NEC Foundation of America, General Motors, Microsoft Corporation, and the National Science Foundation under grant number UA0308065. Any opinions, findings and conclusions or recommendations expressed in this material are those of the authors and do not necessarily reflect those of the National Science Foundation.

\section{REFERENCES}

[1] Anson, D.K. Alternative Computer Access. Philadelphia, PA: F. A. Davis Co., 1997.

[2] Blickenstorfer, C.H. Graffiti: Wow! Pen Computing Magazine, January 1995, 30-31.

[3] Buxton, W., Hill, R. and Rowley, P. Issues and techniques in touch-sensitive tablet input. Proceedings of the ACM Conference on Computer Graphics and Interactive Techniques (SIGGRAPH '85). San Francisco, CA, July 1985, 215-223.

[4] Cook, A.M. and Hussey, S.M. Assistive Technologies: Principles and Practice. St. Louis: C.V. Mosby Co., 1995.

[5] Co-operwrite, Ltd. myText. http://www.my-text.com/

[6] Ehrlich, K. A conversation with Ted Selker. interactions 4 (5), September/October 1997, 34-47.

[7] Enns, N.R.N. and MacKenzie, I.S. Touchpad-based remote control devices. Summary for the ACM Conference on Human Factors in Computing Systems (CHI '98). Los Angeles, April 1998, 229-230.

[8] Farris, J.S., Jones, K.S. and Anders, B.A. Acquisition speed with targets on the edge of the screen: An application of Fitts' Law to commonly used web browser controls. 
Proceedings of the Human Factors and Ergonomics Society 45th Annual Meeting (HFES '01). Minneapolis, October 2001, 1205-1209.

[9] Fichten, C.S., Barile, M., Asuncion, J.V., and Fossey, M.E. What government, agencies, and organizations can do to improve access to computers for postsecondary students with disabilities: Recommendations based on Canadian empirical data. International Journal of Rehabilitation Research 23 (3), 2000, 191-199.

[10] Goldberg, D. and Richardson, C. Touch-typing with a stylus. Proceedings of the ACM Conference on Human Factors in Computing Systems (INTERCHI '93). Amsterdam, January 1993, 80-87.

[11] Guerette, P. and Sumi, E. Integrating control of multiple assistive devices: A retrospective review. Assistive Technology 6 (1), 1994, 67-76.

[12] Hinckley, K., Czerwinski, M. and Sinclair, M. Interaction and modeling techniques for desktop two-handed input. Proceedings of the ACM Symposium on User Interface Software and Technology (UIST '98). San Francisco, November 1998, 49-58.

[13] Isokoski, P. and Kaki, M. Comparison of two touchpadbased methods for numeric entry. Proceedings of the ACM Conference on Human Factors in Computing Systems (CHI '02). Minneapolis, April 2002, 25-32.

[14] Kraus, L.E., Stoddard, S. and Gilmartin, D. Chartbook on Disability in the United States. Washington, D.C.: National Institute on Disability and Rehabilitation Research, 1996.

[15] LoPresti, E.F., Romich, B.A., Hill, K.J. and Spaeth, D.M. Evaluation of mouse emulation using the wheelchair joystick. Proceedings of the 27th Annual Conference of the Rehabilitation Engineering and Assistive Technology Society of North America (RESNA '04). Orlando, Florida, June 2004. Proceedings online at http://www.resna.org/

[16] Ludolph, F. and Perkins, R. The Lisa user interface. Summary for the ACM Conference on Human Factors in Computing Systems (CHI '98). Los Angeles, April 1998, 1819.

[17] MacKenzie, I.S. and Oniszczak, A. A comparison of three selection techniques for touchpads. Proceedings of the ACM Conference on Human Factors in Computing Systems (CHI '98). Los Angeles, April 1998, 336-343.

[18] MacKenzie, I.S. and Soukoreff, R.W. Phrase sets for evaluating text entry techniques. Extended Abstracts of the ACM Conference on Human Factors in Computing Systems (CHI '03). Ft. Lauderdale, Florida, April 2003, 754-755.

[19] Myers, B.A. Using hand-held devices and PCs together. Communications of the ACM 44 (11). New York: ACM Press, 2001, 34-41.

[20] Myers, B.A., Stiel, H., and Gargiulo, R. Collaboration using multiple PDAs connected to a PC. Proceedings of the ACM Conference on Computer Supported Cooperative Work (CSCW '98). Seattle, November 1998, 285-294.

[21] Myers, B.A., Wobbrock, J.O., Yang, S., Yeung, B., Nichols, J. and Miller, R. Using handhelds to help people with motor impairments. Proceedings of the ACM Conference on
Assistive Technologies (ASSETS '02). Edinburgh, Scotland, July 2002, 89-96.

[22] Prentke Romich Company. WiVik On-Screen Keyboard, version 3. http://www.wivik.com/

[23] Rekimoto, J. ThumbSense: Automatic input mode sensing for touchpad-based interactions. Extended Abstracts of the ACM Conference on Human Factors in Computing Systems (CHI '03). Ft. Lauderdale, Florida, April 2003, 852-853.

[24] Romich, B.A., LoPresti, E.F., Hill, K.J., Spaeth, D.M., Young, N.A. and Springsteen, J.P. Mouse emulation using the wheelchair joystick: Preliminary performance comparison using four modes of control. Proceedings of the 25th Annual Conference of the Rehabilitation Engineering and Assistive Technology Society of North America (RESNA '02). Minneapolis, June/July 2002, 106-108.

[25] Shein, F., Hamann, G., Brownlow, N., Treviranus, J., Milner, M. and Parnes, P. WiViK: A visual keyboard for Windows 3.0. Proceedings of the 14th Annual Conference of the Rehabilitation Engineering and Assistive Technology Society of North America (RESNA '91). Kansas City, Missouri, June 1991, 160-162.

[26] Soukoreff, R.W. and MacKenzie, I.S. Metrics for text entry research: An evaluation of MSD and KSPC, and a new unified error metric. Proceedings of the ACM Conference on Human Factors in Computing Systems (CHI '03). Ft. Lauderdale, Florida, April 2003, 113-120.

[27] Spaeth, D.M., Jones, D.K. and Cooper, R.A. Universal control interface for people with disabilities. Saudi Journal of Disability Rehabilitation 4 (3), 1998, 207-214.

[28] Switch-It, Inc. Mouse driver. http://www.switchit-inc.com/pages/Mouse\%20Controls.html

[29] Switch-It, Inc. Touch drive. http://www.switchit-inc.com/pages/Touch\%20Drive.html

[30] Wobbrock, J.O. The benefits of physical edges in gesturemaking: Empirical support for an edge-based unistroke alphabet. Extended Abstracts of the ACM Conference on Human Factors in Computing Systems (CHI '03). Ft. Lauderdale, Florida, April 2003, 942-943.

[31] Wobbrock, J.O., Myers, B.A. and Aung, H.H. Writing with a joystick: A comparison of date stamp, selection keyboard, and EdgeWrite. Proceedings of Graphics Interface (GI '04). Canadian Human-Computer Communications Society. London, Ontario, May 2004, 1-8.

[32] Wobbrock, J.O., Myers, B.A. and Hudson, S.E. Exploring edge-based input techniques for handheld text entry. Third Int'l Workshop on Smart Appliances and Wearable Computing (IWSAWC '03). In Proceedings of the 23rd IEEE Conference on Distributed Computing Systems Workshops (ICDCS '03). Providence, Rhode Island, May 2003, 280282.

[33] Wobbrock, J.O., Myers, B.A. and Kembel, J.A. EdgeWrite: A stylus-based text entry method designed for high accuracy and stability of motion. Proceedings of the ACM Symposium on User Interface Software and Technology (UIST '03). Vancouver, British Columbia, November 2003, 61-70. 